\title{
Validación de una escala para medir la felicidad: un análisis factorial confirmatorio con estudiantes universitarios multiculturales
}

William Oswaldo Flores López ${ }^{1}$

\section{Información de artículo:}

\section{Recibido: 30/09/2019}

Aprobado:30/10/2019

\section{Palabras claves:}

Felicidad, Psicometría,

Validez,

Confiabilidad,

Análisis Factorial Confirmatorio.

\section{Keywords:}

Happiness,

Psychometrics,

Validity,

Reliability,

Confirmatory Factor Analysis.

\section{Resumen}

La felicidad es un estado afectivo de satisfacción plena que experimenta subjetivamente el individuo en posición de un bien anhelado, sin embargo, definir la felicidad, valorarla, medirla o cuantificarla es complicado. Por ello, en este trabajo se presenta la validación de una escala para medir la felicidad. Se ha administrado a 237 estudiantes universitarios procedentes de los pueblos indígenas Miskitos, Indígenas Mayangnas, Creole (Afrodescendientes) y mestizos de las comunidades de la Costa Caribe Nicaragüense. Los resultados muestran buenos ajustes y propiedades psicométricas. La realización de un modelo de ecuaciones estructurales ha evaluado la concurrencia y relación de dos dimensiones relacionado con el fenómeno de la felicidad, encontrando la asociación entre la satisfacción y alegría de vivir; así mismo, la pertinencia de la sub escala sentido positivo de la vida. Estos resultados muestran la idoneidad de ambas sub escalas para evaluar de forma conjunto la felicidad, dada su importante relación y similitud, lo que los convierten en buenas herramientas para las intervenciones psicoeducativas en contextos multiculturales.

\section{Validation of a scale to measure happiness: a confirmatory factor analysis with multicultural university students}

\section{Abstract}

Happiness is an affective state of full satisfaction subjectively experienced by the individual in the position of a desired good, however, defining happiness, valuing it, measuring it or quantifying it is complicated. Therefore, this paper presents the validation of a scale to measure happiness. It has been administered to 237 university students from the Miskito and Mayangnas indigenous peoples,

\footnotetext{
${ }^{1}$ Doctor en Educación. Profesor Investigador de la Universidad de las Regiones Autónomas de la Costa Caribe Nicaragüense. Email: william.flores@uraccan.edu.ni, (1): https://orcid.org/oooo-ooo2-1016-1620
} 
Creole (Afro-descendant) and mestizo of the communities of the Nicaraguan Caribbean Coast. The results show good settings and psychometric properties. The realization of a model of structural equations has evaluated the concurrence and relationship of two dimensions related to the phenomenon of happiness, finding the association between satisfaction and joy of living; likewise, the relevance of the positive sense sub-scale of life. These results show the suitability of both sub-scales to jointly assess happiness, given their important relationship and similarity, which make them good tools for psychoeducational interventions in multicultural contexts.

\section{Introducción}

Existe un exponencial aumento de estudios e intervenciones, que demanda el diseño, validación y confirmación de instrumentos estandarizados que midan la felicidad. Entre ellos, se destaca la escala de la felicidad subjetiva de Lyubomirsky et al. (1999); la escala de felicidad de Pavot et al. (1999); el inventario de felicidad de Lewis et al. (2002); la escala de depresión y felicidad de Joseph et al. (2004); el índice de felicidad de Seligman et al. (2005); y la escala factorial de Alarcón (2006). Estas escalas tratan de medir la felicidad desde componentes compuestos por las dimensiones afectivo positivo y afectivo negativo, así como, mencionan Vera-Villarroel et al. (2010) evalúan un componente cognitivo de satisfacción vital. Desde esta perspectiva, la felicidad se torna vital, por cuanto, todo ser humano anhela ser feliz, sin embargo, definir la felicidad, aún más valorarla, medirla o cuantificarla, es muy complicada (Arias et al., 2016).

Por ello, en este articulo se tiene como objetivo determinar las propiedades psicométricas de validez y confiabilidad de la escala que mide la felicidad de Alarcón (2006) mediante un análisis factorial confirmatorio con estudiantes universitarios multiculturales. La realización de esta investigación se justifica, en primer lugar, a pesar de que la escala que mide la felicidad ha sido usada en diversas poblaciones, no se ha reportado estudios de validación con muestras de poblaciones indígenas, afrodescendientes y mestizas de Nicaragua. En segundo lugar, el empleo de la escala en futuros estudios, en referencia con otras variables, podría ser de utilidad para las intervenciones educativas de las instituciones de educación superior interculturales.

\section{Literatura}

La felicidad es un estado afectivo de satisfacción plena que experimenta subjetivamente el individuo en posición de un bien anhelado. Según Alarcón (2006) se puede distinguir las propiedades de la conducta feliz siguiente: (a) la felicidad es un sentimiento de satisfacción que vivencia una persona, y sola ella, en su vida interior; el componente subjetivo individualiza la felicidad entre las personas; (b) el hecho de ser un estado de la conducta, alude a la estabilidad temporal de la felicidad, puede ser duradera, pero, a la vez, es predecible; (c) la felicidad supone la posesión de un bien, se es feliz en tanto se posee el bien deseado, es el objeto anhelado el que hace 
la felicidad de una persona; (d) el bien o bienes que generan la felicidad son de naturaleza variada; y (e) no se descarta que en un tiempo histórico y en un contexto sociocultural determinado, las aspiraciones colectivas pueden coincidir en desear algún bien o bienes (p. 101).

En este sentido, investigadores como Arraga et al. (2012) realizan un estudio con 633 venezolanos entre 60 y 85 años, estos autores analizaron la confiabilidad de la escala mediante el coeficiente de alfa de Cronbach's con valores entre ítems o,82 y o,84 de fiabilidad, y con una fiabilidad total de o,84. El coeficiente de partición por mitades, con el procedimiento de Spearman-Brown alcanzó una $r=0,89$, mientras que el coeficiente de Guttman $r=0,89$, evidenciando valores altos que respaldan la alta confiabilidad de la escala. En cambio, Toribio et al. (2012) aplicaron el instrumento de la felicidad a 405 estudiantes de preparatoria, mujeres y hombres de 14 a 19 años, comprobando que la escala tiene cuatro factores dimensionales que se pueden explicar en el 50,32\% de la varianza total.

Morillo (2013) realizó el análisis de validez de la escala de felicidad, mediante el método ítem-test con el objetivo de estudiar la correlación entre las respuestas de cada ítem y la puntuación total. El factor sentido positivo de la vida muestra coeficientes de correlaciones entre 0,436 y o,560; en el factor de satisfacción con la vida oscilan entre 0,78 y o,838; en el factor realización personal fluctúan entre o,713 y o,802; y finalmente, en el factor de alegría de vivir, oscilan entre o,869 y o,907. Los coeficientes fiabilidad en los factores son: sentido positivo de la vida de o,88; satisfacción con la vida de o,87; realización personal de o,84; y alegría de vivir de o,9o. Mientras que la escala total presenta un coeficiente alfa de Cronbach's de o,94.

Finalmente, Arias et al. (2016), realizaran un estudio con una muestra conformada por 322 estudiantes universitarios con un rango de edades entre 17 y 28 años, los coeficientes de correlaciones obtenidos oscilan entre o,33 y o,64, el análisis factorial exploratorio alcanzo un valor Kaiser, Meyer y Olkin de o,927, confirmando cuatro factores mediante la técnica de rotación Promax. Los resultados del análisis de fiabilidad evidencian que la confiabilidad de la escala oscila entre o,91 a o,92, en el caso de los cuatros factores de la escala de la felicidad oscilan en rango aceptables y buenos.

En referencia con lo anterior, los diferentes trabajos han reportado resultados concluyentes respecto a los factores asociados a la felicidad, por lo que es deseable el análisis, la validación y confirmación de la estructura factorial de la escala que mide la felicidad en poblaciones en contexto universitarios multiculturales. En este sentido, se ha elegido la escala de la felicidad de Alarcón (2006), debido a su proximidad a la situación de partida: estudiantes inmersos en cursos de formación universitario. 


\section{Metodología de investigación}

\subsection{Diseño}

Esta investigación es de carácter instrumental porque responde a problemas orientados a demostrar las propiedades psicométricas de los instrumentos de medida (Montero \& León, 2007).

\subsection{Participantes}

La muestra utilizada fue de 237 estudiantes universitarios de ambos sexos (Mujeres=141 y Hombres 96) de la Universidad de las Regiones Autónomas de la Costa Caribe Nicaragüense, y con un rango de edad que oscila entre 16 a 36 años $(\mathrm{M}=21,13 ; \mathrm{DT}=1,41)$, procedentes de pueblos indígenas (Miskitos=18; Mayangna=18), afrodescendientes $($ Creole $=6)$ y mestizo $($ Mestizo $=195)$ de las Comunidades de la Costa Caribe de Nicaragua.

Por ello, se seleccionaron las carreras universitarias mediante un muestreo intencionado por accesibilidad, y se contactó con las autoridades del Recinto Universitario Las Minas para invitarles a participar en el estudio. Una vez aceptada la invitación se realizaron entrevista a la secretaría académica para trasladar la intención de realizar un estudio sobre la felicidad y los momentos de aplicación de instrumentos en las aulas de clases. La recogida de datos se realizó por personal del grupo de investigación. Tras explicar a los estudiantes cómo cumplimentar la escala de felicidad, su administración fue individual y duró un máximo de 20 minutos. Se recogieron el mismo día para su posterior análisis de datos.

\subsection{Instrumentos}

Se utilizó la escala factorial para medir la felicidad de Alarcón (2006), que está compuesto por 27 ítems, 11 describen el sentido positivo de la vida (alfa=o,88); 6 satisfacción con la vida (alfa $=79) ; 6$ realización personal (alfa $=76)$; y 4 alegría por vivir $(a l f a=0,72)$. Todos los ítems tienen un diseño tipo Likert, con una puntuación entre 1 a 5 , donde 1 significa totalmente en desacuerdo; y 5 totalmente de acuerdo. 
Tabla 1: Escala para medir la felicidad de Alarcón (2006)

\begin{tabular}{|c|c|c|}
\hline Factores & Indicadores & Ítems \\
\hline $\begin{array}{l}\text { Sentido positivo } \\
\text { de la vida }\end{array}$ & $\begin{array}{l}\text { Percepción y vivencia de libertad; responsa- } \\
\text { bilidad y autodeterminación; cumplimiento } \\
\text { de metas vitales; visión positiva de la vida, } \\
\text { del futuro y de sí mismo; y autorrealización } \\
\text { (García-Alandete, GalleGo-Pérez \& Pérez- } \\
\text { Delgado, 2009). }\end{array}$ & $\begin{array}{l}\text { K26. Todavía no he encontrado Sentido a mi existencia. } \\
\text { K20. La felicidad es para algunas personas, no para mí. } \\
\text { K18. Tengo problemas tan hondos que me quitan la tranquilidad. } \\
\text { K23. Para mí, la vida es una cadena de sufrimientos. } \\
\text { K22. Me siento triste por lo que soy. } \\
\text { Ko2. Siento que mi vida está vacía. } \\
\text { K19. Me siento un fracasado. } \\
\text { K17. La vida ha sido injusta conmigo. } \\
\text { K11. La mayoría del tiempo no me siento feliz. } \\
\text { Ko7. Pienso que nunca seré feliz. } \\
\text { K14. Me siento inútil. }\end{array}$ \\
\hline $\begin{array}{l}\text { Satisfacción con } \\
\text { la vida }\end{array}$ & $\begin{array}{l}\text { La satisfacción con la vida se define como una } \\
\text { evaluación global que la persona hace sobre } \\
\text { su vida Esta definición señala que al realizar } \\
\text { esta evaluación la persona examina los as- } \\
\text { pectos tangibles de su vida, sopesa lo bueno } \\
\text { contra lo malo, lo compara con un estándar } \\
\text { o criterio elegido por ella y llega a un juicio } \\
\text { sobre la satisfacción con su vida (Atienza, } \\
\text { Pons, Balaguer \& Merita, 2000). }\end{array}$ & $\begin{array}{l}\text { K04. Estoy satisfecho con mi vida. } \\
\text { K03. Las condiciones de mi vida son excelentes. } \\
\text { K05. La vida ha sido buena Conmigo. } \\
\text { K01. En la mayoría de las cosas, mi vida está cerca de mi ideal. } \\
\text { K06. Me siento satisfecho con lo Que soy. } \\
\text { K10. Me satisfecho porque estoy donde tengo que estar. }\end{array}$ \\
\hline $\begin{array}{l}\text { Realización } \\
\text { personal }\end{array}$ & $\begin{array}{l}\text { El concepto de realización personal guarda } \\
\text { correspondencia con la definición de felicidad } \\
\text { que supone la orientación del individuo hacia } \\
\text { metas que considera valiosas para su vida. Por } \\
\text { ello, se mide la: autosuficiencia, autarquía, } \\
\text { tranquilidad emocional, placidez; todas son } \\
\text { condiciones para conseguir el estado de } \\
\text { felicidad completa (Pérez, Fuentes, Medina, } \\
\text { Escobar \& Van Barneveld, 2012). }\end{array}$ & $\begin{array}{l}\text { K24. Me considero una persona realizada. } \\
\text { K21. Estoy satisfecho con lo que hasta ahora he alcanzado. } \\
\text { K27. Creo que no me falta nada. } \\
\text { K25. Mi vida transcurre plácidamente. } \\
\text { K09. Si volviera a nacer no cambiaría casi nada de mi vida. } \\
\text { K08. Hasta ahora he conseguido las cosas que para mí son impor- } \\
\text { tantes. }\end{array}$ \\
\hline Alegría de Vivir & $\begin{array}{l}\text { Referido a ver las experiencias de la vida en } \\
\text { forma positiva y sentirse generalmente bien } \\
\text { (Barrios, \& Villarroel, 2012). }\end{array}$ & $\begin{array}{l}\text { K12. Es maravillo vivir. } \\
\text { K15. Soy una persona optimista } \\
\text { K16. He experimentado la alegría de vivir. } \\
\text { K13. Por lo general, me siento bien }\end{array}$ \\
\hline
\end{tabular}

\subsection{Procedimiento y análisis de los datos}

En cuanto a los análisis estadísticos realizados para alcanzar el objetivo de este estudio se ha empleado análisis factorial de componentes principales utilizando rotación Varimax: previamente se realizaron la prueba de Kaiser, Meyer y Olkin (KMO); y la prueba de esfericidad de Bartlett. Por otra parte, se realizó el análisis factoriales confirmatorios (AFC) para someter a contraste a la estructura factorial de la escala de la felicidad, así como, la realización de un modelo de ecuaciones estructurales para 
contrastar la relación e influencia entre sentido positivo de la vida; satisfacción con la vida; realización personal; y alegría de vivir.

Teniendo de referencia con lo anterior, se asumieron las recomendaciones de $\mathrm{Hu}$ y Bentler (1999), en las cuales un modelo ajusta adecuadamente a los datos observados cuando el estadístico chi-cuadrado en comparación con sus grados de libertad presenta un ratio inferior a cinco, así como, RMSEA (Root Mean Squeare Error of Approximation), SRMR (Standardized Root Mean Square Residual) cuyos valores recomendados son < 0.08, los índices), IFI (Incremental Fit Index) y CFI (Comporative Fit Index), cuyos valores recomendables son >0,95 (Jöreskog y Sörbom, 1993). Como método de estimación se utilizó el de máxima verosimilitud robusta, que permite utilizar correlaciones policóricas, cuyo uso es más idóneo en variables con las características señaladas de altos índices de normalidad y curtosis multivariante y con clara naturaleza ordinal (Flora y Curran, 2004).

La fiabilidad de las dimensiones del modelo y del total de la estructura factorial propuesta fueron examinadas mediante el cálculo de alfa de Cronbach y omega de McDonald's. El programa utilizado para el análisis estadístico fue jamovi versión 1.1.7.o.

\section{Resultados}

Una primera aproximación a la dimensionalidad del constructo se ha realizado un análisis de componentes principales (ACP). Para ello la escala factorial que mide la felicidad fue sometido a la prueba de Kaiser-Meyer-Olkin que alcanzo una puntuación de o,884. La prueba de esfericidad ofreció resultados que indicaban que el análisis era pertinente con un Chi-cuadrado de 2129,646 con una significación de o,ooo. Siguiendo el criterio de Kaiser-Meyer-Olkin, se obtuvieron 2 factores cuyo valor propio es inferior a uno. Los factores extraídos explican el 58,84\% de la varianza total de los datos. Es importante resaltar que el índice KMO pone de manifiesto la adecuación del tamaño muestral utilizada, mientras que la prueba de esfericidad de Bartlett indica que las correlaciones entre ítems no configuran una mera matriz de identidad. Además, se cálculo el determinante de la matriz de correlaciones, cuyo valor fue prácticamente o $(\mathrm{D}=8.139 \mathrm{E}-5)$.

Partiendo de la reducción de la escala factorial, se procedió a realizar un análisis de fiabilidad mediante el alfa de Cronbach's y el omega de McDonald's, encontrado un alfa de $86 \%$ y omega de $87 \%$ en la puntuación total de la escala. En el caso de la fiabilidad ítem a ítem se alcanzó un alfa de Cronbach's ( $\alpha=0,84 a \alpha=0,86)$; y omega de McDonald's ( $\omega=0,86$ a $\omega=0,87$ ). Además, se determinó la homogeneidad de la escala mediante la correlación ítem-escala, los coeficientes de correlación se ubican entre 0,39 a o,59, lo que indica que los ítems discriminan bastante bien. Por otra parte, se realizó un análisis estadístico descriptivo: media ( $M=4,23$ a $M=3,05)$, desviación estándar 
( $D S=1,28$ a $D S=1,04)$; y varianza $(S=1,65$; a $S=1,09)$. Estos resultados confirman el proceso de validación de la escala para medir la felicidad (ver Tabla 2).

Tabla 2: Estadísticos Descriptivos

\begin{tabular}{|c|c|c|c|c|c|c|c|}
\hline K & Ítems del cuestionario & M & DS & $S$ & $\mathrm{r}_{\mathrm{it}}$ & a & $\omega$ \\
\hline $\mathrm{K}_{3}$ & Las condiciones de mi vida son excelentes. & 3.59 & 1.04 & 1.09 & 0,39 & 0,84 & 0,86 \\
\hline $\mathrm{K}_{4}$ & Estoy satisfecho con mi vida. & 4.02 & 1.06 & 1.13 & 0,58 & 0,85 & 0,86 \\
\hline $\mathrm{K}_{5}$ & La vida ha sido buena Conmigo. & 3.84 & 1.10 & 1.21 & 0,49 & 0,86 & 0,86 \\
\hline K6 & Me siento satisfecho con lo Que soy. & 4.10 & 1.04 & 1.09 & 0,44 & 0,86 & 0,86 \\
\hline $\mathrm{K}_{7}$ & Pienso que nunca seré feliz. & 3.81 & 1.22 & 1.49 & 0,40 & 0,86 & 0,86 \\
\hline K11 & La mayoría del tiempo no me siento feliz. & 3.57 & 1.17 & 1.38 & 0,48 & 0,86 & 0,86 \\
\hline K12 & Es maravillo vivir. & 4.23 & 1.04 & 1.10 & 0,49 & 0,86 & 0,86 \\
\hline $\mathrm{K}_{13}$ & Por lo general, me siento bien & 3.77 & 1.05 & 1.11 & 0,50 & 0,86 & 0,86 \\
\hline $\mathrm{K}_{14}$ & Me siento inútil. & 4.00 & 1.22 & 1.49 & 0,50 & 0,86 & 0,86 \\
\hline K17 & La vida ha sido injusta conmigo. & 3.63 & 1.25 & 1.57 & 0,51 & 0,85 & 0,86 \\
\hline K18 & Tengo problemas tan hondos que me quitan la tranquilidad. & 3.40 & 1.26 & 1.60 & 0,50 & 0,86 & 0,86 \\
\hline K19 & Me siento un fracasado. & 4.11 & 1.08 & 1.16 & 0,59 & 0,85 & 0,86 \\
\hline $\mathrm{K}_{20}$ & La felicidad es para algunas personas, no para mí. & 4.00 & 1.14 & 1.30 & 0,54 & 0,85 & 0,86 \\
\hline$K_{22}$ & Me siento triste por lo que soy. & 4.06 & 1.08 & 1.17 & 0,57 & 0,85 & 0,86 \\
\hline$K_{23}$ & Para mí, la vida es una cadena de sufrimientos. & 3.92 & 1.18 & 1.40 & 0,56 & 0,85 & 0,86 \\
\hline$K_{26}$ & Todavía no he encontrado Sentido a mi existencia. & 3.73 & 1.24 & 1.55 & 0,43 & 0,86 & 0,86 \\
\hline $\mathrm{K}_{27}$ & Creo que no me falta nada & 3.05 & 1.28 & 1.65 & 0,32 & 0,86 & 0,87 \\
\hline
\end{tabular}

Lo anterior, permitió calcular la matriz de correlaciones, con correlaciones que oscilan entre el o,63 y o,03 (Ver Figura 1). 


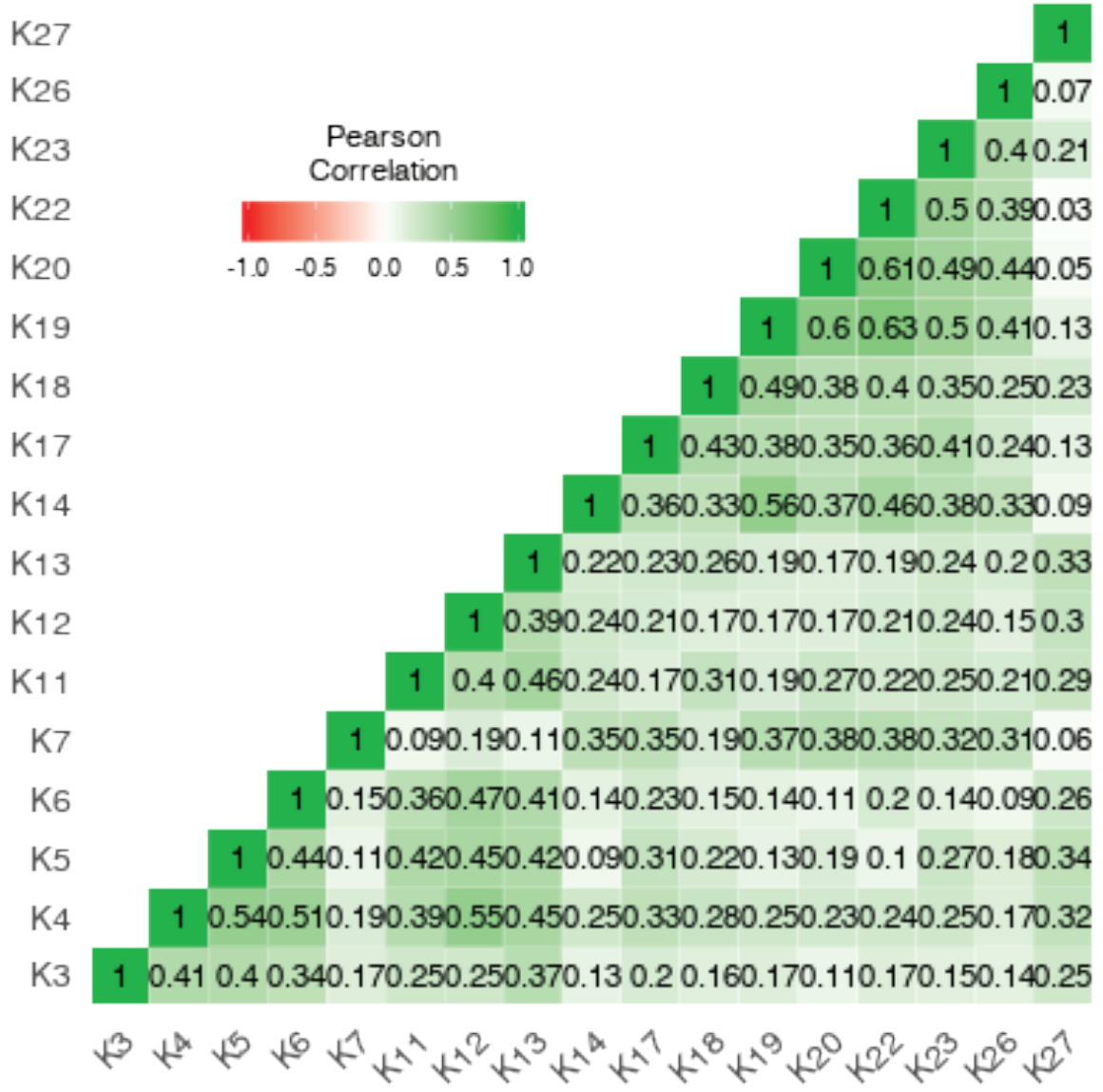

Figura 1: Matriz de correlaciones entre los ítems de la escala

Para validar y confirmar la reducción factorial, se desarrollo el análisis factorial confirmatorio (AFC). La solución factorial más adecuada (ver figura 2) muestra dos factores correlacionados con un ajuste optimo (SAV: Satisfacción y Alegría de Vivir; y SV: Sentido positivo de la Vida) con unos índices de ajuste con valores $x^{2}=171, d f=118$; $p=0,001 ; C F I=0,96 ; I F I=0,95 ; R M S E A=0,043 ; S R M R=0,05$. 


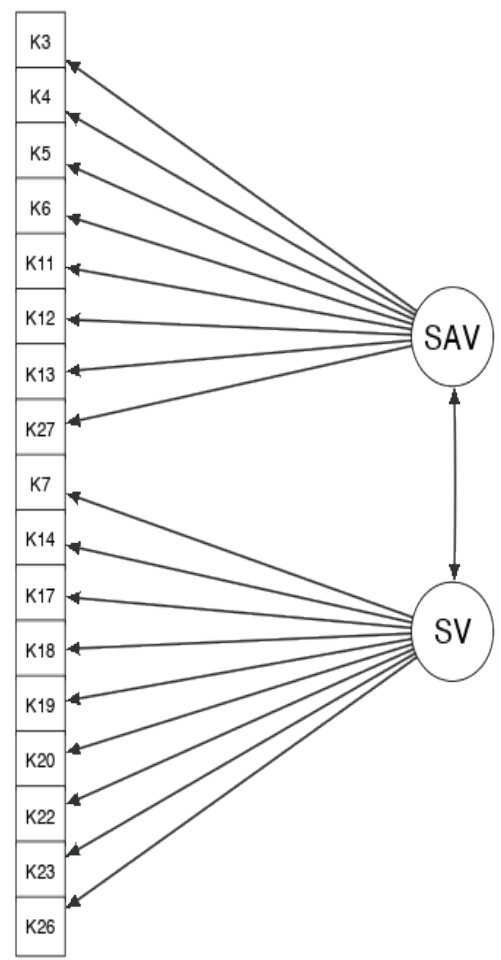

Figura 2: Solución factorial de la escala de la felicidad

También, se cálculo la fiabilidad de los factores resultantes. El factor satisfacción y alegría de vivir alcanzó una confiabilidad de un alfa de Cronbach's de o,83; y un omega de McDonald's de o,84. En cambio, sentido positiva de la vida obtuvo un alfa de Cronbach's de o,85 y un omega de McDonald's de o,86.

\section{Discusión y resultados}

En esta investigación se ha tratado determinar las propiedades psicométricas de validez y confiabilidad de la escala que mide la felicidad de Alarcón (2006). Respecto con dicho objetivo, puede expresarse que los resultados son muy positivos. Desde esta perspectiva, se encontró que la prueba de Kaiser-Meyer-Olkin muestra una puntuación de $K M O=0,884$ con una significación de $p=0,000$, este resultado se aproxima al estudio de Arias et al. (2016) que alcanzó un $K M O=0,927$. Además, el análisis factorial de componentes principales realizado en la escala que mide de la felicidad se configura en dos factores dimensionales y 17 ítems explicado en un $58,84 \%$ de la varianza total de los datos, debatiendo los resultados de Toribio et al. (2012) que muestra que la escala tiene cuatro factores dimensionales que se pueden explicar en el 50,32\% de la varianza total, así como, el estudio de Arias et al. (2016), confirma la existencia cuatro 
factores a través del análisis factorial exploratorio mediante la técnica de rotación Promax con una cantidad de varianza explicada del 61,94\%.

En este contexto, la escala factorial que mide la felicidad muestra la adecuación, validez y confiabilidad de las dos dimensiones principales de este fenómeno y pueden describirse en un solo modelo, que incluye correlación entre ambas subescalas tal y como se ha ido hallando en estudios previos (Alarcón, 2006; Arraga et al., 2012; Toribio et al., 2012; Morillo, 2013; Arias et al., 2016). Se podría decir que la totalidad de los participantes perciben el fenómeno de la felicidad desde una doble dimensión satisfacción y alegría de vivir, y sentido positivo de la vida, que se muestra de forma empírica con buenos ajuste resultado los valores $x^{2}=171, d f=118 ; p=0,001 ; C F I=0,96$; $I F I=0,95 ; R M S E A=0,043 ; S R M R=0,05$. Conjuntamente, con correlaciones que oscilan entre el o,63 y 0,03 .

En el caso de la homogeneidad de la escala se ubica entre o,39 a 0,59, lo que indica que los ítems de la escala discriminan bastante bien, coincidiendo con los estudios Kline (1998), Hogan (2004) y Arias et al. (2016). Así, los coeficientes de correlación pueden ser catalogados como moderados y altos (Delgado et al., 2006; y Arias et al., 2016). Lo anterior explica que los 17 ítems miden indicadores de un mismo constructo, esto permite coadyuvar con eficacia a su medición. Por otra parte, se realizó un análisis estadístico descriptivo obteniendo puntuaciones medias ( $M=4,23$ a $M=3,05)$, desviación estándar $(D S=1,28$ a $D S=1,04)$; y varianza $(S=1,65 ; a S=1,09)$. En definitiva, estos resultados obtenidos no pueden considerarse estrictamente concluyentes porque las respuestas a los ítems del instrumento se encuentran íntimamente asociadas con los participantes del estudio, lo cual altera las relaciones entre los ítems y la prueba (Domínguez et al., 2014).

En relación con la confiabilidad de la escala se encontró un índice de fiabilidad alfa de $86 \%$ y omega de $87 \%$ en la puntuación total de la escala. En el caso de la fiabilidad ítem a ítem se alcanzó un alfa de Cronbach's ( $\alpha=0,84 a \alpha=0,86)$; y omega de McDonald's $(\omega=0,86 a \omega=0,87)$. Estos resultados superan las magnitudes de $85 \%$ reportados por DeVellis (2012) considerados como muy alto por Campo-Arias et al., (2008), pero se aproximan con los logrados por el estudio de Alarcón (2006) que alcanzo índices de fiabilidad entre 0,72 a 0,88, así como, los conseguidos por Arias et al. (2006) que logró un índice entre 0,90 a 0,91 de fiabilidad.

En definitiva, pensando en la mejora de la practica didáctica y pedagógica socioeducativa, el disponer de instrumentos confiables y empíricamente contrastado cobra importancia en la investigación sobre el fenómeno de la felicidad en estudiantes universitarios multiculturales, teniendo de referencia que las principales fuentes de felicidad son el hacer el bien a los demás y el disponer en práctica las habilidades y fortalezas (Seligman, 2006). Además, el desarrollo de nuevos instrumentos debe basarse en una cuidadosa consideración de las ventajas y desventajas de los que ya 
existen (Berne et al., 2013), en este sentido, esta validación aporta lo siguiente: a) la posibilidad de medir y evaluar en un mismo instrumento el fenómeno de la felicidad; y b) este nuevo cuestionario son un aportación al campo de estudio ya que es muy reducido el número de instrumento que han sido sometidos a un riguroso análisis empírico y han sido replicado mediante análisis factorial confirmatorio para contrastar sus fortalezas y debilidades.

\section{Lista de referencias}

Alarcón, Reynaldo (2006). Desarrollo de una Escala Factorial para Medir la Felicidad. Revista Interamericana de Psicología/Interamerican Journal of Psychology, 40(1), 96-106.

Arias, W., Caycho, T., Ventura, J., Maquera, C., Ramírez, M., \& Tamayo, X. (2016). Análisis exploratorio de la escala de felicidad de Lima en universitarios de Arequipa (Perú). Psychologia: Avances de la Disciplina, 10(1), 13-24.

Arraga, M. V., \& Sánchez, M. (2012). Validez y confiabilidad de la escala de Felicidad de Lima en adul- tos mayores venezolanos. Universitas Psychologica, 11(2), 381-393.

Atienza, F. L., Pons, D., Balaguer, I., \& Merita, M. G. (2000). Propiedades psicométricas de la Escala de Satisfacción con la Vida en adolescentes. Psicothema, 12(2), 314-319.

Berne, S., Frisén, A., Schultze-Krumbholz, A., Scheithauer, H., Naruskov, K., Luik, P., . . Zukauskiene, R. (2013). Cyberbullying assessment instruments: A systema- tic review. Aggression and Violent Behavior, 18, 320-334. http://doi. org/10.1016/j.avb.2012.11.022

Campo-Arias, A., \& Oviedo, H. C. (2008). Revisión/Review propiedades psicométricas de una escala: la consistencia interna. Revista de Salud Pública, 10, 831-839.

DeVellis, R. F. (2012). Scale Development, Theory and Applications. Los Angeles: SAGE.

Dominguez, S.A., Villegas, G., \& Centeno, S.B. (2014). Procastinación académica: validación de una escala en una muestra de estudiantes de una universidad privada. Liberabit, 20(2), 293-304.

Flora, D. B. y Curran, P. J. (2004). An Empirical Evaluation of Alternative Methods of Estimation for Confirmatory Factor Analysis With Ordinal Data. Psychological Methods, 9, 466-491. http://dx.doi.org/10.1037/1082-989X.9.4.466 
García-Alandete, J., GalleGo-Pérez, J. F., \& Pérez-Delgado, E. (2009). Sentido de la vida y desesperanza: un estudio empírico. Universitas Psychologica, 8(2), 447-454.

Hogan, T. (2004). Pruebas psicológicas. Una introducción práctica. México D.F.: Manual Moderno.

Hu, L. y Bentler, P. M. (1999). Cutoff criteria for fit indexes in cova- riance structure analysis: Conventional criteria versus new alternati- ves. Structural Equation Modeling: A Multidisciplinary Journal, 6, 1-55. http://dx.doi. org/10.1080/10705519909540118

Jöreskog, K. G. y Sörbom, D. (1993). Lisrel 8: Structural Equation Modeling with the Simplis Command Language. Chicago, IL: Scientific Software International.

Joseph, S., Linley, P. A., Harwood, J., Lewis, C.A., \& McCollam, P. (2004). Rapid assessment of well-being: The short depression-happiness scale (SDHS). Psychology and Psychotherapy: Theory, Research and Practice, 77, 463-478.

Kline, P. (1998). The new psychometrics: science, psychology and measurement. London: Routhledge.

Lyubomirsky, S., \& Lepper, H. S. (1999). A measure of subjective happiness: Preliminary reliability and construct validation. Social Indicators Research, 46, 137-155.

Montero, I., \& León, O. G. (2007). Guía para nombrar los estudios de investigación en Psicología. International Journal of Clinical and Health Psychology, 7(3), 847-862.

Pavot, W. \& Diener. (1999). Review of the satisfaction with life scale. Psychological Assessment, 5, 164-172.

Pérez, L. T., Fuentes, N. I. G. A. L., Medina, J. L. V., Escobar, S. G., \& Van Barneveld, H. O. (2012). Validación de la Escala de Felicidad de Alarcón para adolescentes mexicanos. Psicología Iberoamericana, 20(1), 71-79.

Seligman, M. E. P., Steen, T. A., Park, N., \& Peterson, C. (2005). Positive psychology progress empirical validation of interventions. American Psychologist, 60, 410-421.

Toribio, L., González, N. I., Valdez, J. L., González, S., \& Van Berneveld, H. O. (2012). Validación de la Escala de Felicidad de Alarcón para adolescentes mexicanos. Psicología Iberoamericana, 20(1), 71-79.

Vera-Villarroel, P., Buela-Casal, G., Zych, I., Córdova-Rubio, N., Celis-Atenas, K., Zepeda, L., \& Spielberger, C. D. (2010). Chilean experimental version of the state-trait 
depression questionnaire (ST-DEP): state subscale (S-DEP). Psychological Reports, 106, 65-77.

Vera-Villarroel, P., Celis-Atenas, K., \& Córdova-Rubio, N. (2011). Evaluación de la felicidad: análisis psicométrico de la escala de felicidad subjetiva en población chilena. Terapia psicológica, 29(1), 127-133. 